\title{
INEQUALITIES FOR GENERAL MATRIX FUNCTIONS
}

\author{
BY MARVIN MARCUS ${ }^{1}$ AND HENRYK MINC ${ }^{2}$
}

Communicated by A. S. Householder, November 22, 1963

I. Introduction. In [4] Schur proved the following beautiful result. If $H$ is a subgroup of the symmetric group of degree $m, S_{m}$, and $\chi(\sigma)$ is a character of degree 1 of $H$, then

$$
\operatorname{det} A \leqq \sum_{\sigma \in H} \chi(\sigma) \prod_{t=1}^{m} a_{t \sigma(t)}
$$

for any $m$-square positive semi-definite hermitian matrix $A$. Observe that if $H$ is the identity group, the inequality (1) is the Hadamard determinant theorem $\operatorname{det} A \leqq \prod_{i=1}^{m} a_{i i}$. In [3] it was conjectured that per $A \geqq \prod_{i=1}^{m} \quad a_{i i}$ and in [2] this inequality was proved. Here per $A=\sum_{\sigma \in S_{m}} \prod_{t=1}^{m} a_{t \sigma(t)}$ is the permanent of $A$.

The purpose of the present paper is to announce some inequalities for the general matrix function

$$
d_{\chi}(A)=\sum_{\sigma \in H} \chi(\sigma) \prod_{t=1}^{m} a_{t \sigma(t)} .
$$

We shall see subsequently that Schur's inequality (1) is an immediate corollary to our Theorem 4.

\section{Main results.}

THEOREM 1. If $N$ is m-square normal with characteristic roots $\eta_{1}, \cdots, \eta_{m}$, then

$$
\left|d_{x}(N)\right| \leqq \frac{1}{m} \sum_{i=1}^{m}\left|\eta_{i}\right|^{m}
$$

In case $\chi \equiv 1$, we have the following generalization of the van der Waerden conjecture in the non-negative hermitian case $[3 ; 5]$.

TheOREM 2. Let $A$ be an $m$-square positive semi-definite hermitian. Let the ith row sum of $A$ be denoted by $r_{i}, i=1, \cdots, m$, and suppose $\sum_{i=1}^{m} r_{i}=r>0$. Then

I The work of this author was supported by the National Science Foundation under grant G. P. 1085.

${ }^{2}$ The work of this author was supported by the Air Force Office of Scientific Re search. 


$$
d_{1}(A) \geqq h \prod_{i=1}^{m}\left|r_{i}\right|^{2} / r^{m}
$$

where $h$ is the order of the group $H$. Equality holds in (4) if and only if either (i) $A$ has a zero row, or (ii) $\rho(A)=1$, where $\rho(A)$ is the rank of $A$.

Let $Q_{m, n}$ be the totality of strictly increasing sequences $\omega$ of length $m$ chosen from $1, \cdots, n, 1 \leqq \omega_{1} \leqq \cdots \leqq \omega_{m} \leqq n$. If $\omega$ and $\tau$ are any two sequences of length $m$ chosen from $1, \cdots, n$, then $A[\omega \mid \tau]$ is the $m$-square matrix whose $(i, j)$ entry is $a_{\omega_{i}, r_{j}}, i=1, \cdots, m$, $j=1, \cdots, m$. In case $\omega, \tau \in Q_{m, n}$ then $A[\omega \mid \tau]$ is an $m$-square submatrix of $A$.

Theorem 3. Let $A$ be an $n$-square positive semi-definite hermitian matrix with characteristic roots $\alpha_{1} \geqq \alpha_{2} \geqq \cdots \geqq \alpha_{n}$. Then

$$
\prod_{i=1}^{m} \alpha_{n-i+1} \leqq d_{\chi}(A[\omega \mid \omega]) \leqq \frac{1}{m} \sum_{i=1}^{m} \alpha_{i}^{m} .
$$

Theorem 4. If $A$ is $m \times n$ and $B$ is $n \times m$, then

$$
\left|d_{x}(A B)\right|^{2} \leqq d_{x}\left(A A^{*}\right) d_{x}\left(B^{*} B\right) .
$$

In case $\chi \equiv 1$ equality holds in (6) only if (i) $A$ has a zero row, or (ii) $B$ has a zero column, or (iii) $A=D P B^{*}$ where $D$ is a diagonal matrix and $P$ is a permutation matrix.

This result without any discussion of equality is found in [3]. Schur's result can now be stated.

Corollary 1. If $A$ is an m-square positive semi-definite hermitian, then

$$
\operatorname{det} A \leqq d_{\chi}(A) .
$$

This inequality is easily proved from (6) as follows. Let $A=X^{*} X$ where $X$ is an $m$-square triangular matrix. Then

$$
\begin{aligned}
\operatorname{det} A & =\operatorname{det} X^{*} X=\operatorname{det} X \operatorname{det} X^{*} \\
& =d_{x}(X) d_{x}\left(X^{*}\right)=d_{x}(I X) d_{x}\left(X^{*} I\right) \\
& \leqq\left(d_{x}\left(X^{*} X\right)\right)^{1 / 2}\left(d_{x}\left(X^{*} X\right)\right)^{1 / 2}=d_{x}(A) .
\end{aligned}
$$

Let $\Gamma_{m, n}$ denote the set of $n^{m}$ sequences $\omega=\left(\omega_{1}, \cdots, \omega_{m}\right), 1 \leqq \omega_{i} \leqq n$, $i=1, \cdots, n$, and define an equivalence relation in $\Gamma_{m, n}$ by $\omega \sim \tau$ if and only if there exists a $\sigma \in H$ such that $\omega^{\sigma}=\left(\omega_{\sigma(1)}, \cdots, \omega_{\sigma(m)}\right)$ $=\left(\tau_{1}, \cdots, \tau_{m}\right)=\tau$. For $\omega \in \Gamma_{m, n}$ let $\nu(\omega)$ be the number of $\sigma \in H$ for which $\omega^{\sigma}=\omega$. By $\Delta$ we shall denote a fixed system of distinct representatives for the equivalence relation. For example, if $H=S_{m}$ we 
can choose $\Delta$ to be the set of $C_{m, n+m-1}$ nondecreasing sequences $\gamma, \gamma_{1} \leqq \cdots \leqq \gamma_{m}$.

The following result underlies Theorem 1 and is of some interest in itself.

Theorem 5 (Generalized Cauchy-Binet expansion). Let $A$ be $m \times n$ and $B$ be $n \times m$ matrices. Then

$$
d_{\boldsymbol{\chi}}(A B)=\sum_{\gamma \in \Delta} \frac{1}{\nu(\gamma)} d_{\boldsymbol{x}}(A[1, \cdots, m \mid \gamma]) d_{\chi}(B[\gamma \mid 1, \cdots, m]) .
$$

It is well known that certain relations must obtain between subdeterminants of a matrix (the quadratic relations). It is a useful fact that in the case of a unitary matrix a related result is true for the general function $d_{x}$. For each $t=1, \cdots, n$ and $\gamma \in \Gamma_{m, n}$ let $m_{t}(\gamma)$ denote the multiplicity of occurrence of the integer $t$ in $\gamma$.

THEOREM 6. If $m=n$ and $U$ is an $n$-square unitary matrix, then for each $t=1, \cdots, n$

$$
\sum_{\gamma \in \Delta} \frac{m_{t}(\gamma)}{\nu(\gamma)}\left|d_{x}(U[\gamma \mid 1, \cdots, n])\right|^{2}=1 .
$$

A matrix is called doubly stochastic if every row and column sum is 1 . Generalizing what is currently known about the van der Waerden conjecture we have as an immediate consequence of Theorem 2:

Corollary 2. Let $A$ be an m-square doubly stochastic positive semidefinite hermitian matrix. Then, if $h$ is the order of $H$,

$$
d_{1}(A) \geqq \frac{h}{m^{m}} .
$$

Equality holds in (10) if and only if $A=J_{m}$, the matrix all of whose entries are $1 / \mathrm{m}$.

To see this, simply set each $r_{i}=1$ and $r=m$ in (4). The equality can hold if and only if every row of $A$ is a multiple of the first row. Since each row sum is 1 it follows that all the rows are identical, say $\left(a_{11}, \cdots, a_{1 m}\right)$. Since the $j$ th column sum is 1 it follows that $a_{1 j}=1 / m$ and hence $A=J_{m}$.

COROLlARy 3. If $A$ is an m-square matrix with singular values $\alpha_{1} \geqq \cdots \geqq \alpha_{m}$, then

$$
\left|d_{x}(A)\right|^{2} \leqq \frac{1}{m} \sum_{i=1}^{m} \alpha_{i}^{2 m}
$$


In case $\chi \equiv 1$ the equality holds in (11) if and only if $A=D P$ where $D$ is a diagonal matrix each of whose main diagonal entries have the same absolute value and $P$ is a permutation matrix corresponding to a permutation in $H$.

This follows directly from (6) and (3).

Corollary 4. Let $N$ be an m-square normal matrix and let $A=N N^{*}$ $=N^{*} N$. Let $A^{1 / 2}$ denote the unique positive semi-definite determination of the square root of $A$. Then

$$
\left|d_{\chi}(N)\right| \leqq d_{\chi}\left(A^{1 / 2}\right) \text {. }
$$

If $\chi \equiv 1$ and (12) is equality, then $N$ is of the form $D P A^{1 / 2}$ where $D$ is a diagonal matrix and $P$ is a permutation matrix.

CoRollary 5. If $N$ is an m-square doubly stochastic, normal and has non-negative entries, then

$$
\text { per } N \leqq \frac{\rho(N)}{m}
$$

The inequality is strict unless either $N$ is a permutation matrix or $m=2$ and $N=J_{2}$.

The characteristic roots of $N$ do not exceed 1 in modulus and exactly $\rho(N)$ of them are nonzero. Thus (13) follows immediately from (3). Now suppose (13) is equality. Then every nonzero characteristic root of $N$ is of modulus 1 . By the Perron-Frobenius theorem [1] we obtain $P$ and $Q$, permutation matrices, such that $P N Q$ is a direct sum of primitive matrices [1]. The moduli of the characteristic roots of $N$ and $P N Q$ are the same and $\rho(N)=\rho(P N Q)$. Thus each of the primitive main diagonal blocks in $P N Q$ has precisely one characteristic root equal 1 , the rest 0 . Thus $P N Q$ is a direct sum of matrices $J_{m_{i}}, i=1, \cdots, r, m_{i} \geqq 2, i=1, \cdots, r$, together with an $h$-square identity matrix: $r=\rho(N)-h, \rho(N) \geqq h \geqq 0$. Suppose $r>0$. Then

$$
\text { per } N=\operatorname{per} P N Q=\prod_{i=1}^{r} \frac{m_{i} !}{m_{i}^{m_{i}}}=\frac{\rho(N)}{m}=\frac{h+r}{h+\sum_{i=1}^{r} m_{i}} \geqq \frac{r}{\sum_{i=1}^{r} m_{i}} \geqq \frac{r}{\prod_{i=1}^{r} m_{i}} \text {. }
$$

Hence

$$
\prod_{i=1}^{r} m_{i} ! \geqq r \prod_{i=1}^{r} m_{i}^{m_{i}-1}
$$

This implies $r=1, m_{1}=2$ and $P N Q=I_{m-2}+J_{2}$. But then per $P N Q=\frac{1}{2}$ 
while $\rho(P N Q) / m=(m-1) / m$. Thus $m=2$. If $r=0, N$ is clearly a permutation matrix.

CoRollary 6. If $A$ is an m-square doubly stochastic matrix with nonnegative entries, then

$$
\text { per } A \leqq\left(\frac{\rho(A)}{m}\right)^{1 / 2} \text {. }
$$

Equality holds in (14) if and only if $A$ is a permutation matrix.

This follows from (13) and the fact that per $A \leqq\left(\operatorname{per}\left(A^{*} A\right)\right)^{1 / 2}[3]$.

III. Method of proof. Let $V$ be an $n$-dimensional unitary space with inner product $(x, y)$ and let $V^{(m)}$ be the tensor product of $V$ with itself $m$ times. For $x_{i} \in V, i=1, \cdots, m$, define the symmetry operator

$$
T\left(x_{1} \otimes \cdots \otimes x_{m}\right)=\sum_{\sigma \in H} \chi(\sigma) x_{\sigma^{-1}(1)} \otimes \cdots \otimes x_{\sigma^{-1}(m)} .
$$

Then $T^{2}=h T, T^{*}=T$ where $h$ is the order of the subgroup $H$. Set $x_{1} * \cdots * x_{m}=T\left(x_{1} \otimes \cdots \otimes x_{m}\right)$ and observe that

$$
\left(x_{1} * \cdots * x_{m}, y_{1} * \cdots * y_{m}\right)=h d_{x}(A)
$$

where $a_{i j}=\left(x_{i}, y_{j}\right)$. The inner product in (15) is the standard one in $V^{(m)},\left(x_{1} \otimes \cdots \otimes x_{m}, y_{1} \otimes \cdots \otimes y_{m}\right)=\prod_{i=1}^{m}\left(x_{i}, y_{i}\right)$. It turns out that Theorem 5 is a restatement of Parseval's Theorem in the symmetry class of tensors $T\left(V^{(m)}\right)$. We then apply (8) to a matrix of the form $U^{*} D U$ where $D$ is diagonal and $U$ is unitary to obtain Theorem 6 . We can prove the inequality (3) as follows. Let $N$ $=U^{*} \operatorname{diag}\left(\eta_{1}, \cdots, \eta_{m}\right) U$ and set $c_{\gamma}=\left|d_{x}(U[\gamma \mid 1, \cdots, m])\right|^{2}$ for $\gamma \in \Delta$. Then from Theorem 5

$$
\begin{aligned}
\left|d_{\chi}(N)\right| & =\left|\sum_{\gamma \in \Delta} \frac{c_{\gamma}}{\nu(\gamma)} \prod_{t=1}^{m} \eta_{t}^{m_{t}(\gamma)}\right| \leqq \sum_{\gamma \in \Delta} \frac{c_{\gamma}}{\nu(\gamma)} \prod_{t=1}^{m}\left|\eta_{t}\right|^{m_{t}(\gamma)} \\
& \leqq \sum_{\gamma \in \Delta} \frac{c_{\gamma}}{\nu(\gamma)} \mid \frac{\left.\sum_{t=1}^{m} m_{t}(\gamma)\left|\eta_{t}\right|\right|^{m}}{m} \\
& \leqq \sum_{\gamma \in \Delta} \frac{c_{\gamma}}{\nu(\gamma)} \frac{1}{m} \sum_{t=1}^{m} m_{t}(\gamma)\left|\eta_{t}\right|^{m} \\
& =\frac{1}{m} \sum_{t=1}^{m}\left|\eta_{t}\right|^{m} \sum_{\gamma \in \Delta} \frac{m_{t}(\gamma) c_{\gamma}}{\nu(\gamma)}=\frac{1}{m} \sum_{t=1}^{m}\left|\eta_{t}\right|^{m}
\end{aligned}
$$


The remaining results can be proved by similar techniques. Thus inequality (4) is obtained by projecting a decomposable element of the symmetry class $T\left(V^{(m)}\right)$ onto a suitable tensor and using the Cauchy-Schwarz inequality.

The discussion of the cases of equality requires special and somewhat involved arguments.

\section{REFERENCES}

1. F. R. Gantmacher, The theory of matrices. II, Chelsea, New York, 1959.

2. Marvin Marcus, The permanent analogue of the Hadamard determinant theorem, Bull. Amer. Math. Soc. 69 (1963), 494-496.

3. Marvin Marcus and Morris Newman, Inequalities for the permanent function, Ann. of Math. (2) 75 (1962), 47-62.

4. I. Schur, Über endliche Gruppen und Hermitesche Formen, Math. Z. 1 (1918), 184-207.

5. B. L. van der Waerden, Aufgabe 45, J.-Ber. Deutsch. Math. Verein. 35 (1926), 117.

University of California, Santa Barbara 